Proceedings

\title{
Piezoelectric Sensor Signal Analysis after Interface Changes between the Sensor and the Structure under Monitoring ${ }^{\dagger}$
}

\author{
Pedro Giroto 1,*, Paulo R. Aguiar ${ }^{1}$, Felipe A. Alexandre ${ }^{1}$, Pedro Oliveira Junior ${ }^{1}$, \\ Martin Aulestia Vieira ${ }^{1}$, Eduardo Carlos Bianchi ${ }^{2}$ and Erick Ruas ${ }^{1}$ \\ 1 Department of Electrical Engineering, São Paulo State University, Av. Eng. Luiz Edmundo Carrijo Coube, \\ 14-01, Bauru 17033-360, Brazil; aguiarpr@feb.unesp.br (P.R.A.); felipe.alexandre@feb.unesp.br (F.A.A.); \\ pedroliveira931@gmail.com (P.O.J.); martin.aulestia@unesp.br (M.A.V.); erick.ruas@unesp.br (E.R.) \\ 2 Department of Mechanical Engineering, São Paulo State University, \\ Av. Eng. Luiz Edmundo Carrijo Coube, 14-01, Bauru 17033-360, Brazil; bianchi@feb.unesp.br \\ * Correspondence: pedrocgiroto@gmail.com; Tel.: +55-19-996236880. \\ + Presented at the 6th International Electronic Conference on Sensors and Applications, \\ 15-30 November 2019; Available online: https://ecsa-6.sciforum.net/.
}

Published: 14 November 2019

\begin{abstract}
This study aims to show the influences of the sensor installation interface in the industrial environment. This contribution is focused on analyzing the response behavior of piezoelectric transducers subjected to successive installations, using digital signal processing and non-destructive structural health monitoring (SHM) techniques. Tests were performed to simulate the installation conditions of a piezoelectric sensor, which was coupled to a holder carrying a steel body and submitted to successive reinstallations. Different signals were obtained for each installation, and the results can bring initial elucidations on the subject and pave the way for future studies.
\end{abstract}

Keywords: piezoelectric diaphragm; monitoring; digital signal processing

\section{Introduction}

Machining processes are an essential part of component manufacturing and in most cases require high precision and quality surface finish, thus adding high value to the workpiece. However, to achieve this quality, the settings of all parts, including tools, machines, temperature, and parameters must be correct. The monitoring of these processes through electrical signals and sensors arise as an alternative to control the variables that influence the quality of the final product.

In the industrial environment, sensors are widely used to perform various monitoring functions. Piezoelectric transducers are cheap, lightweight, low power, present a linear response under a low electric field, and have an important property to convert mechanical energy into electrical energy, and vice versa; this property is known as the piezoelectric effect [1]. The piezoelectric effect provides an electromechanical coupling with the part, allowing to evaluate the conditions of the part by the electrical response of the transducer.

Ultrasonic waves are widely used in non-destructive monitoring techniques and produced by piezoelectric transducers. Ultrasonic waves are waves that have frequencies of more than $20 \mathrm{kHz}$ and have some characteristics, such as length, speed, frequency, pressure and period. The interaction of these waves with the propagation medium results in two phenomena: the first is change in velocity and the second is the attenuation of the wave, both due to the mechanisms of absorption and dispersion of the material media [2]. 
Regardless of the sensor chosen, its installation and the coupling up to the surface of a material present imperfections, called "coupling errors". These errors can create a "coupling effect", which cannot be completely eliminated, but should be limited as much as possible [3]. In this way, every time a sensor is reinstalled, new "coupling errors" may appear, changing the system interface. Also in [3], it is observed that the sensor design is related to coupling errors, and there is essentially a direct relationship between the sensitive structure design and the coupling effect.

In the study of da Silveira et al. [4], an analysis was carried out on different methods of installation of piezoelectric transducers (direct installation, magnetic coupling and using aluminum foils) and their influence on the damage detection in structures through SHM techniques. The results show that the conventional method of installation is the most sensitive for lower frequencies, while the other methods are more sensitive for higher frequencies.

In this context, one of the aspects studied in the works of [5-7] is the use of coupling substances between the sensor and the part under analysis. According to Colombo et al. [5], a good coupling effect is necessary to obtain good data, and for this the sensor surface should be clean and the coupler material should be thin and fill the entire contact surface and remove air gaps. The nature of the substance used affects the quality of the coupling and the reliability of the signal obtained.

According to Theobald et al. [6], the couplers used in their study improve the transmission between the contact surface and the sensor, but they add more variability to the process. Different couplers may have different transmission properties, affecting the apparent sensitivity and frequency response of the sensor. Thus, when choosing a coupler, there are several factors to be taken into account, such as the quality of the signal received, adaptability to the surface, durability, ease of installation and reinstallation, and repeatability, among others [5]. In Fasana and Garibaldi [7], several types of couplers were tested, such as greases, waxes, oils and adhesives, and it was observed that even improving the sensor response, each one brings a variability to the system and has defects.

The present work aims to study the influence of the reinstallation of the piezoelectric sensor and seek repeatability in the signals; however, the air was used as a coupler in this investigation. Future works related to this theme can explore the use of different couplers. Also, this work seeks to show that these differences between the signals are related to the change of interface of the piezoelectric transducer installation. The performance analysis of the analyzed transducer was performed by spectral analysis of the responses obtained from the sensors, as well as the identification of the frequencies most sensitive to the coupling changes of the analyzed sensor.

\section{Material and Methods}

The analyzed sensor is a buzzer (PZT-lead zirconate titanate), which consists of $12 \mathrm{~mm}$ brass diameter and $9 \mathrm{~mm}$ piezoelectric ceramic. This sensor was placed into a metal casing with a magnet. Subsequently, the sensor was coupled to the holder used to fix parts in the surface grinding that served as the medium transmission through magnetic coupling, allowing the mobility of the transducer and enabling the process of evaluating the influence of the installation interface. It is worth mentioning that the interface between the sensor and the holder was established without a couplant.

In order to evaluate the frequency response of the analyzed sensor, a chirp signal was used and emitted through another PZT transducer glued to the holder. Thus, this PZT receives the chirp signal and transmits the waves (piezoelectric effect) through the holder until it reaches the analyzed sensor (reverse piezoelectric effect). This technique was presented by [8] and it was named "Chirp Through Transmission".

The LabVIEW software was used for generating the chirp signals and a data acquisition board, DAQ NI USB-6221, was used for sending these signals to the glued PZT. The chirp signals contained frequencies from 0 to $250 \mathrm{kHz}$ and amplitudes of $5 \mathrm{~V}$. For the analysis, five chirp packages were sent to the glued sensor. Thus, the excitation signal consists in these five chirp packages. This excitation signal was sent three times in order to verify the repeatability of the measures and prevent errors. A ScopeCorder model DL850, from Yokogawa company (Tokyo, Japan) was responsible for acquiring the received signal from the analyzed PZT. The sample rate used was $2 \mathrm{MS} / \mathrm{s}$. The setup used in the tests is presented in Figure 1. 


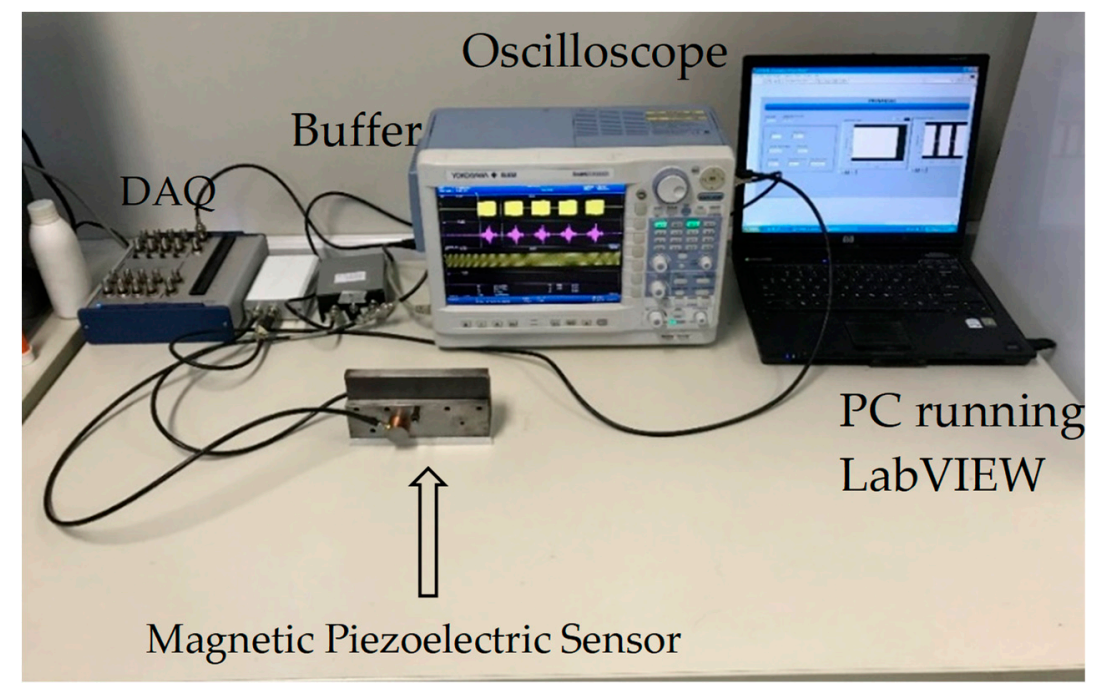

Figure 1. Experimental Setup.

Five installations of the analyzed sensor were performed to verify the influence of reinstalling the sensor without a couplant. Each test consisted of the following steps: (a) fixing the magnetic PZT in the holder at a position marked with a pencil on the holder, which was used to approximately maintain the sensor reinstallation at the same position; (b) sending the excitation signal to the glued sensor; (c) acquiring and storing the received signal of the magnetic PZT by the oscilloscope. The reinstallation influence was studied for two different positions of the analyzed sensor in the holder. For the first position, the magnetic sensor was fixed in the opposite side of the holder in relation to the glued transducer. For the second position, the magnetic sensor was fixed on the same side of the holder in relation to the glued transducer.

In relation to signal processing, fifteen spectra were calculated from the chirp packages that were repeated three times. Then, the mean spectrum of these spectra was computed. This procedure was performed for each of the five reinstallations of the transducer. Then, the overall mean spectrum was obtained from the spectra of the five installations in order to study the influence of interface change between the sensor and structure being monitored.

In order to evaluate the variations of the analyzed sensor's frequency response in each installation, the percentage error between these responses and the overall mean spectrum was computed. Equation (1) presents the percentage error for a given frequency band, where $x_{o}$ is the overall mean spectrum, $x$ is the spectrum of one of the five installations, $\omega_{o}$ is the initial frequency and $\omega_{f}$ is the final frequency.

$$
\operatorname{Error}(\%)=\sum_{\omega_{o}}^{\omega_{f}} \frac{\left|x_{o}-x\right|}{x_{o}} * 100
$$

It is important to mention that the error was computed for frequency bands of $10 \mathrm{kHz}$ throughout the whole spectrum, which allowed to identify the bands that presented the greatest changes in frequency response after the reinstallation of the sensor.

\section{Results and Discussion}

Figure 2a shows the chirp signal emitted by the DAQ and Figure $2 b$ the signal received by the analyzed transducer. It is observed that the received signal is attenuated considerably, but it still has enough energy to perform the analysis. Besides the attenuation, it is observed that some frequencies of the emission signal were transmitted with greater effectiveness. These differences are partially due to the properties of the material (holder) in which the sensors were coupled, since the composition of the materials changed the propagation of waves [9]. In addition, the waves emitted behave differently with their frequencies as well as certain factors such as reflection, dispersion, refraction among others. 


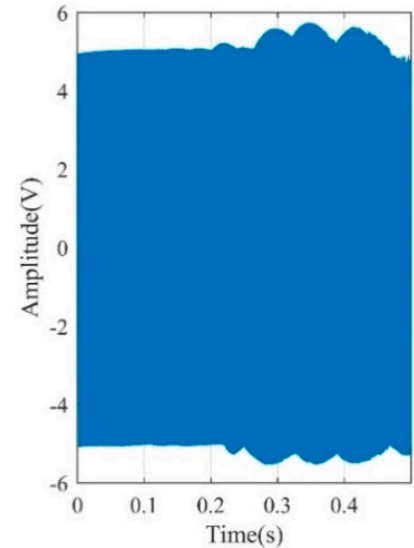

(a)

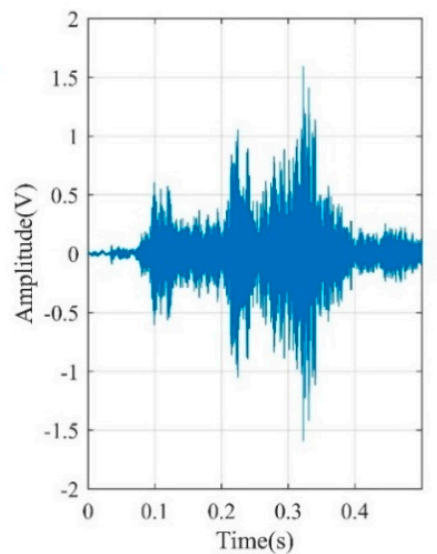

(b)

Figure 2. (a) Signal emitted by the DAQ; (b) signal received by the receiver PZT transducer.

Figure 3a shows the frequency spectra of the five installation tests performed for the first position considered. It is noted that the behavior of the signals is similar throughout the spectrum and that in some frequency bands, as in the magnification shown in Figure 3, there is a significant amplitude difference. Figure $3 b$ shows the frequency spectrum for the second position tests of the receiving transducer. The analysis is analogous to Figure 3a, where a similar behavior can also be observed between the curves, but there are point variations in certain frequencies. These differences are related to the reinstallation of the sensor.

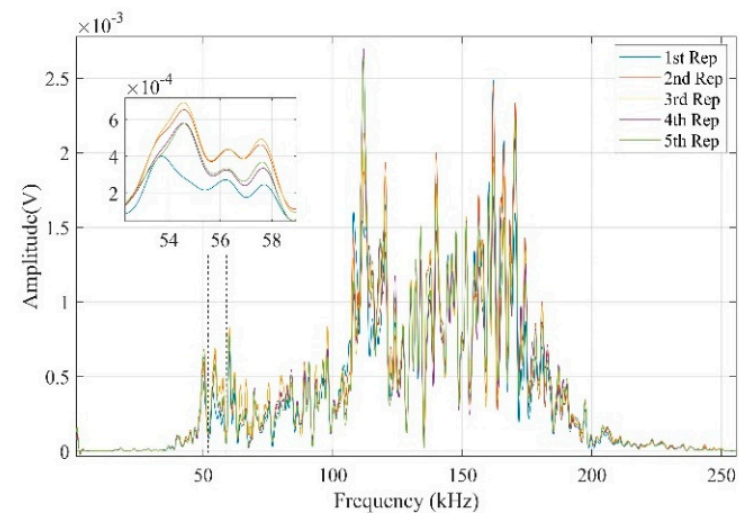

(a)

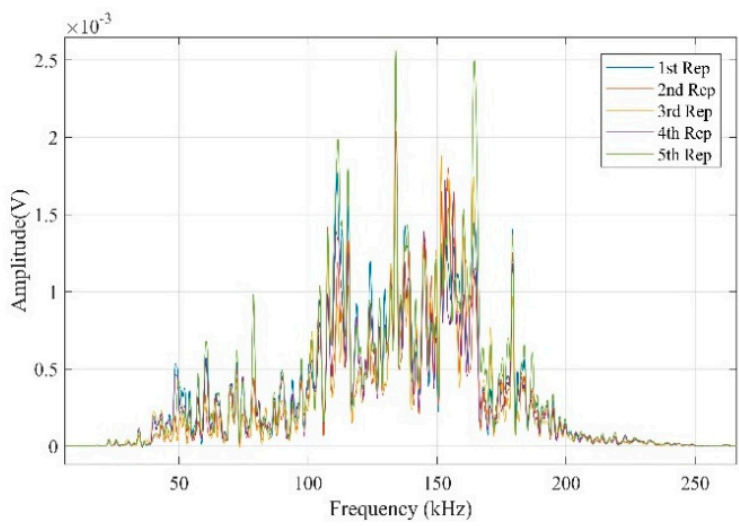

(b)

Figure 3. (a) Frequency spectrum of the chirp signals sent in the first position; (b) frequency spectrum of the chirp signals sent in the second position.

Figure 4 shows the percentage error between the overall mean spectrum and each test performed, and in each frequency band analyzed, in position 1 (Figure 4a) and in position 2 (Figure 4b). The abscissa has 25 bands, being the first from 0 to $10 \mathrm{kHz}$, the second from $10 \mathrm{kHz}$ to $20 \mathrm{kHz}$, and so on. Each color in the graphs of Figure 4 represents a specific frequency band. As expected, there are errors in every band, related to the reinstallation. Some bands are more sensible, for example, band number $8(70 \mathrm{kHz}$ to $80 \mathrm{kHz})$ for Figure $4 \mathrm{a}$, and band number 5 (40 kHz to $50 \mathrm{kHz})$ for Figure $4 \mathrm{~b}$.

Figure 5 shows the mean percentage error between the five tests in each frequency band analyzed. For the analysis of Figure 5, it is first important to relate it to Figure 3, because it is observed that in the first and last frequency bands, up to $40 \mathrm{kHz}$ and after $200 \mathrm{kHz}$ approximately, the frequency signal has very low amplitude, where small variations are more influential. Thus, the correct analysis is to consider only bands between $40 \mathrm{kHz}$ and $200 \mathrm{kHz}$. Then, the bands that are in this frequency range are the ones from 5 to 20 of Figure 5 . 


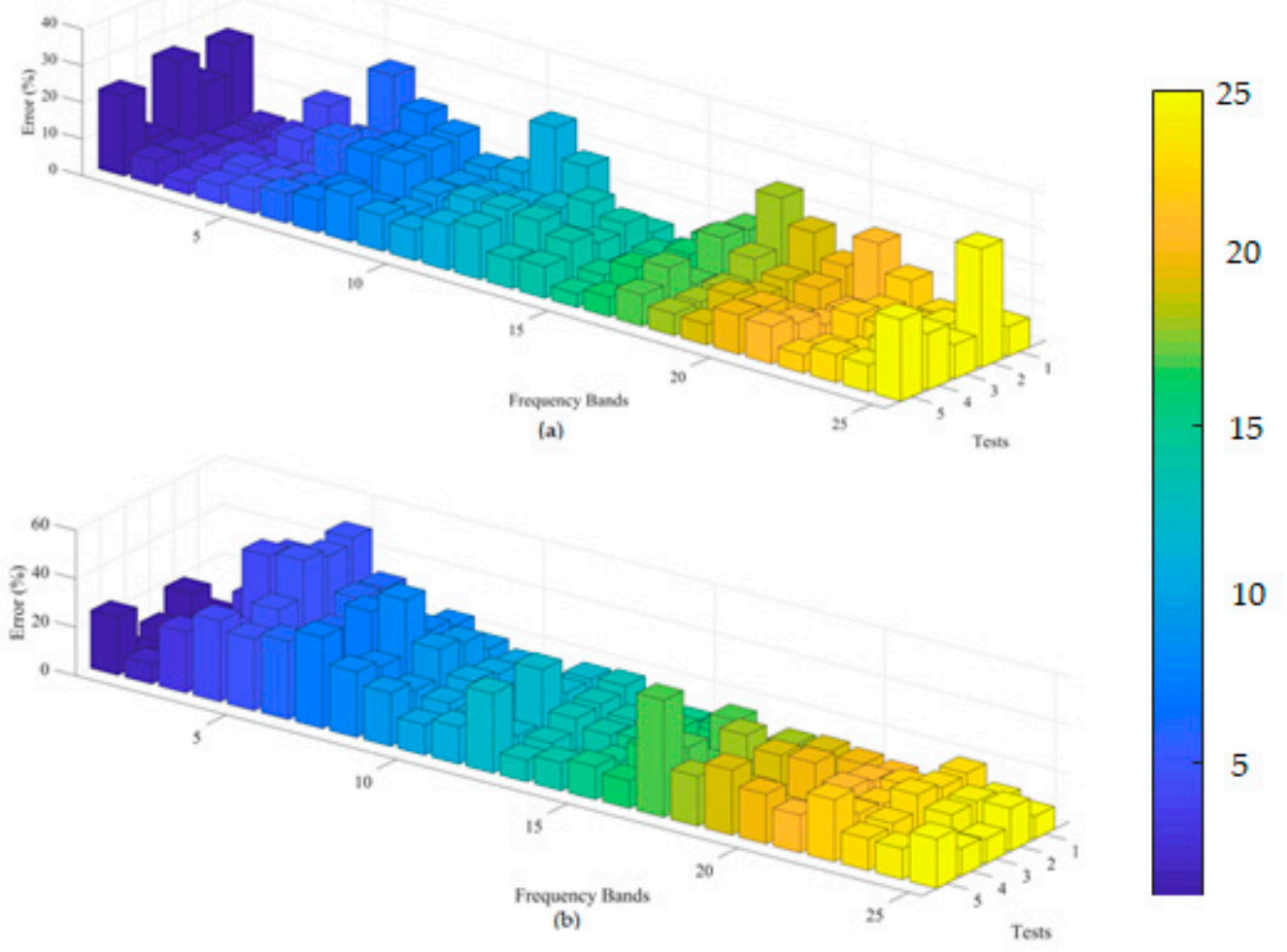

Figure 4. (a) Percentage error related to the frequency bands of position 1 ; (b) percentage error related to the frequency bands of position 2.

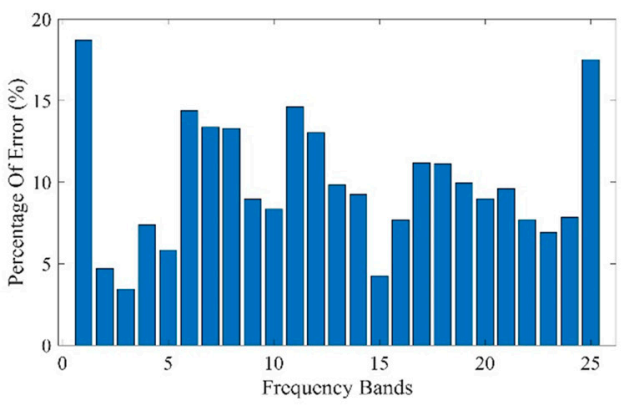

(a)

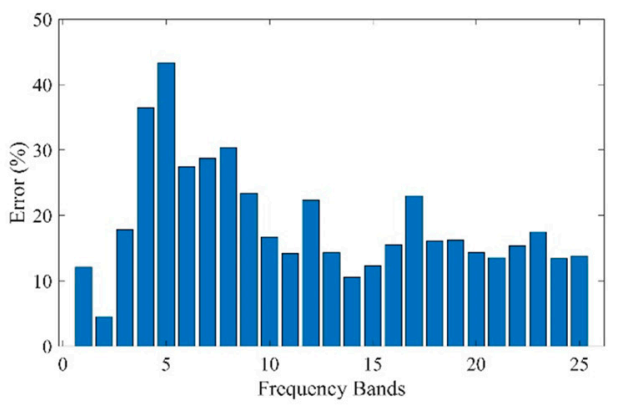

(b)

Figure 5. (a) Average of the percentage errors related to the frequency bands of position 1; (b) average of the percentage errors related to the frequency bands of position 2.

The next point to be highlighted is that in both positions, the percentage error was high and remained somewhat constant, apart from some bands. In Figure $5 \mathrm{a}$, which is related to position 1, the mean percentage error was below $10 \%$, with bands 6 to $8(50 \mathrm{kHz}$ to $80 \mathrm{kHz})$ and 11 to $12(100 \mathrm{kHz}$ to $120 \mathrm{kHz}$ ) standing out and reaching close to $15 \%$ of percentage error. In Figure $5 \mathrm{~b}$, which is related to position 2 , the mean percentage error was greater than position 1 , with more than $15 \%$ on average, and with bands $5(40 \mathrm{kHz}$ to $50 \mathrm{kHz})$ standing out and reaching more than $40 \%$ of percentage error, and bands 6 to 8 ( $50 \mathrm{kHz}$ to $80 \mathrm{kHz}$ ) reaching more than $25 \%$.

After this analysis, the first conclusion that is obtained, is that of position 1, in which the analyzed transducer on the opposite side of the transmitter had, in general, less percentage error than position 2, where the analyzed transducer was next to the transmitter. This may be related to the size of the path traveled by the wave and its travel time, but this is a topic to be discussed in other work.

The focus of this work is, from the analysis of the frequency bands, to identify the bands that best characterize and are influenced by the reinstallations of the transducer. As previously said, in position 1, the bands with the greatest percentage error were from 6 to $8(50 \mathrm{kHz}$ to $80 \mathrm{kHz})$ and from 
11 to $12(100 \mathrm{kHz}$ to $120 \mathrm{kHz})$, and in position 2, the bands 5 to $8(40 \mathrm{kHz}$ to $80 \mathrm{kHz}$ ) were highlighted. It is observed that the intersection between the two positions, in bands 6 to 8 ( $50 \mathrm{kHz}$ to $80 \mathrm{kHz}$ ), indicated a possible frequency range of greater interference of the reinstallations.

Following the same standard of analysis, there are also bands that stood out when presenting the smallest percentage error in each of the positions. In position 1, band $15(140 \mathrm{kHz}$ to $150 \mathrm{kHz})$ was the one that presented the smallest error, with about $5 \%$. In position 2 , the bands that had less percentage error were from 14 to $15(130 \mathrm{kHz}$ to $150 \mathrm{kHz}$ ) with about 10 to $13 \%$, but these bands did not stand out much, because there were several bands with about $15 \%$ percentage error in this position.

\section{Conclusions}

This work sought to present the influences of changes in the interface installation of sensors in the industrial environment, which can occur due to occasional maintenance, which removes and replaces the sensor in the same place. It has been possible to detect the existence of these differences, which can be named "coupling errors".

From the results obtained, it can be stated that coupling errors have a great influence on the signals received after the transmission of ultrasonic waves. These variations should be considered in the reinstallations of these sensors. In this work, it was sought to identify bands that are more strongly related to the sensitivity of the sensor due to its reinstallation. According to the results, the bands from $50 \mathrm{kHz}$ to $80 \mathrm{kHz}$ presented the biggest coupling errors for the two sensor positions studied, while the band from $140 \mathrm{kHz}$ to $150 \mathrm{kHz}$ presented the smallest error. These findings indicate that these bands may be the ones with the greatest and smallest influence of reinstallations, respectively, and should be the focus of future work, for further clarification and proof of this approach.

Acknowledgments: The authors would like to thank the Sao Paulo Research Foundation (FAPESP), under grant \#2017/18148-5 and the National Council for Scientific and Technological Development (CNPQ), under grant 306435/2017-9 for supporting this research work.

\section{References}

1. Baptista, F.G.; Filho, J.V. Optimal Frequency Range Selection for PZT Transducers in Impedance-Based SHM Systems. IEEE Sens. J. 2010, 10, 1297-1303.

2. Awad, T.S.; Moharram, H.A.; Shaltout, O.E.; Asker, D.; Youssef, M.M. Applications of ultrasound in analysis, processing and quality control of food: A review. Food Res. Int. 2012, 48, 410-427.

3. Akbari, H.; Kazerooni, A. Improving the coupling errors of a Maltese cross-beams type six-axis force/moment sensor using numerical shape-optimization technique. Measurement 2018, 126, 342-355.

4. da Silveira, R.Z.M.; Campeiro, L.M.; Baptista, F.G. Analysis of Sensor Installation Methods in Impedancebased SHM Applications. Procedia Eng. 2016, 168, 1751-1754.

5. Colombo, S.; Giannopoulos, A.; Forde, M.C.; Hasson, R.; Mulholland, J. Frequency response of different couplant materials for mounting transducers. NDT E Int. 2005, 38, 187-193.

6. Theobald, P.; Zeqiri, B.; Avison, J. Couplants and Their Influence on AE Sensor Sensitivity. J. Acoustic Emission 2008, 26, 91-97.

7. Fasana, A.; Garibaldi, L. Measurement of Acoustic Emission Signals: Influence of the Couplant. Key Eng. Mater. 2007, 347, 375-380.

8. Alexandre, F.A.; Aguiar, P.R.; Götz, R.; Aulestia Viera, M.A.; Lopes, T.G.; Bianchi, E.C. A Novel Ultrasound Technique Based on Piezoelectric Diaphragms Applied to Material Removal Monitoring in the Grinding Process. Sensors 2019, 19, 3932.

9. Alexandre, F.; Aguiar, P.R.; Götz, R.; Fernandez, B.O.; Lopes, W.N.; Viera, M.A.A.; Bianchi, E.C.; D’addona, D. Damage detection in grinding of steel workpieces through ultrasonic waves. MATEC Web Conf. 2018, 249,02002 . 